\title{
物 \\ DESENVOLVIMENTO DE UM MODELO DINÂMICO DE MONITORAMENTO DA CARGA NO BASQUETEBOL DE ALTO RENDIMENTO
}

\author{
DEVELOPMENT OF A DYNAMIC LOAD MONITORING MODEL IN HIGH \\ PERFORMANCE BASKETBALL
}

\section{DESARROLLO DE UN MODELO DINÁMICO DE MONITOREO DE CARGA EN BALONCESTO DE ALTO RENDIMIENTO}

\author{
Bruno Teobaldo Campos ${ }^{1}$ \\ Paulo Alberto de Paula ${ }^{2}$ \\ José Ricardo Claudino Ribeiro ${ }^{3}$ \\ Cláudio Olívio Vilela Lima ${ }^{4}$
}

\begin{abstract}
Resumo: No basquetebol as respostas de fadiga aguda e crônica impactam no desempenho e planejamento do treinamento a longo prazo. Dessa forma, o objetivo do presente estudo é demonstrar o monitoramento longitudinal das variáveis de carga e recuperação durante a temporada competitiva de uma equipe profissional e propor um modelo dinâmico de análise e tomada de decisão para o planejamento do treinamento. Os atletas foram submetidos a um monitoramento diário da carga durante 34 semanas. O monitoramento foi composto por variáveis neuromusculares, fisiológicas e perceptivas. A partir de uma análise de regressão múltipla, identificamos uma significância estatística da influência da carga e recuperação fisiológica no desempenho neuromuscular. A partir disso, foi proposto um modelo dinâmico de interpretação da carga e recuperação que permite ajustes diários no planejamento.
\end{abstract}

Palavra-chave: Basquetebol. Monitoramento da Carga. Variabilidade da Frequência Cardíaca. Salto com Contramovimento. Carga Interna.

\footnotetext{
Abstract: In basketball, acute and chronic fatigue responses have an affect on performance and long-term training planning. Thus, the objective of the present study is to demonstrate the longitudinal monitoring of the load and recovery variables during the competitive season of a professional team and to propose a dynamic model of analysis and decision making for training planning. Athletes were monitored daily for 34 weeks. The monitoring measures consisted of neuromuscular, physiological and perceptual variables.

1 Mestre em ciências do esporte (UFMG) e fisiologista do Minas Tênis Clube (MTC). (bruno.campos@minastc.com.br)

${ }^{2}$ Especialista em fisiologia e nutrição esportiva (Passo 1 Uberlândia) e preparador físico de basquetebol do Minas Tênis Clube (MTC). (paulo.alberto@minastc.com.br)

${ }^{3}$ Mestre em ciências da educação (FMH Lisboa) e gerente multidisciplinar do Minas Tênis Clube (MTC). (jose.ricardo@minastc.com.br)

${ }^{4}$ Mestre em ciências do esporte (UFMG) e coordenador das ciências do esporte do Minas Tênis Clube (MTC). (claudio.olivio@minastc.com.br)
} 
From a multiple regression analysis, we identified a statistical significance of the influence of load and physiological recovery on neuromuscular performance. From this, it was proposed a dynamic model of load and recovery interpretation that allows daily adjustments in planning.

Keywords: Basketball. Monitoring training load. Heart rate variability. Counter movement jump. Internal load.

Resumen: En el baloncesto, las respuestas de fatiga aguda y crónica afectan el rendimiento y la planificación del entrenamiento a largo plazo. Por lo tanto, el objetivo del presente estudio es demostrar el monitoreo longitudinal de las variables de carga y recuperación durante la temporada competitiva de un equipo profesional y proponer un modelo dinámico de análisis y toma de decisiones para la planificación del entrenamiento. Los atletas fueron monitoreados diariamente durante 34 semanas. Las medidas de monitoreo consistieron en variables neuromusculares, fisiológicas y perceptivas. A partir de un análisis de regresión múltiple, identificamos una significación estadística de la influencia de la carga y la recuperación fisiológica en el rendimiento neuromuscular. A partir de esto, se propuso un modelo dinámico de interpretación de carga y recuperación que permite ajustes diarios en la planificación.

Palabras clave: Baloncesto. Monitoreo de carga de entrenamiento. Variabilidad de la frecuencia cardíaca. salto de contraataque. Carga interna.

\section{Introdução}

O basquetebol é considerado um esporte intermitente de alta intensidade que requer a utilização do metabolismo aeróbico e anaeróbico, no qual o metabolismo aeróbico predomina durante as ações de baixa intensidade, enquanto o metabolismo anaeróbico (lático e alático) se mostra importante durante as ações de alta intensidade essenciais para um desempenho de sucesso no jogo (CASTAGNA et al., 2010; HOFFMAN et al., 2000; NARAZAKI et al., 2009). As ações de alta intensidade durante o jogo dependem da resposta neuromuscular dos membros inferiores, demonstrada pela elevada quantidade de saltos por jogo, cerca de, $44 \pm 7$ saltos ao longo da partida (BEN ABDELKRIM et al., 2007; PLIAUGA et al., 2015) e podendo chegar a 105 sprints em uma partida oficial, percorrendo de 952 a 1.329 metros em altas velocidades (TAYLOR et al., 2017). Nesse sentido, uma partida de basquetebol pode ocasionar respostas de fadiga agudas e crônicas que perturbam o processo recuperativo.

Essas respostas pós-jogo são inerentes ao processo de treinamento e precisam ser contempladas no planejamento e nas tomadas de decisão. A aplicação da carga de treinamento ocasiona também respostas de fadiga agudas e crônicas, que podem influenciar o desempenho e aumentar o risco de lesões. Um estudo prévio que investigou a relação da carga de treinamento com lesões em atletas de alto rendimento mostrou que picos de variações semanais superiores a $15 \%$ da carga interna $(\mathrm{CI})$ podem representar um aumento no risco de lesões em atletas 
(GABBETT, 2016).

Como explicitado acima, faz-se necessário um processo de registro e monitoramento das respostas fisiológicas da carga de treinamento com propósitos de se alcançar o melhor rendimento esportivo. Com avanços na tecnologia a partir do início da década de 1980 (monitores de frequência cardíaca por telemetria, analisadores bioquímicos portáteis, analisadores de gases respiratórios, analisadores de movimento - GPS), a comunidade científica conseguiu fornecer melhores biomarcadores das respostas fisiológicas e mecânicas durante os treinamentos e as competições (FOSTER et al., 2017), como creatina quinase, imunoglobulinas, relação testosterona-cortisol, concentração de amônia, ureia, variabilidade da frequência cardíaca, entre outros (CARDINALE \& VARLEY, 2017; HEIDARI et al., 2018; LEE et al., 2017).

Nesse contexto, tem-se utilizado o monitoramento da resposta do sistema nervoso autônomo (SNA) através da variabilidade da frequência cardíaca (VFC) a fim de controlar a recuperação e adaptação utilizando uma ferramenta não invasiva (BUCHHEIT, 2014), rápida e imparcial em relação ao avaliador e ao avaliado. A VFC descreve matematicamente as oscilações dos intervalos entre batimentos cardíacos consecutivos (intervalos R-R) e depende do controle do SNA sobre o nódulo sinusal (TASK FORCE, 1996). A VFC se relaciona com a fadiga mental (MIZUNO et al., 2011; 2014), com respostas ao treinamento de força (PANISSA et al., 2016), com o treinamento aeróbico (NAKAMURA et al., 2005), com a adaptação à carga de treinamento em esportes coletivos (NAKAMURA et al., 2015) e com a recuperação à carga de treino (BUCHHEIT et al., 2007; CATALDO et al., 2016; NAKAMURA et al., 2016). Além da identificação da associação entre a VFC e respostas agudas à carga de treinamento, já foram encontradas evidências de maior evolução do desempenho aeróbico em atletas que modularam a carga de treinamento a partir das respostas de VFC (VESTERINEN et al., 2016). Entretanto, a utilização da VFC como ferramenta de controle da recuperação às variações da carga de treinamento não responde adequadamente a todas as situações, e discute-se se esta variável é capaz de identificar todas as formas de manifestação da fadiga (BELLENGER et al., 2016) e se pode ser utilizada como variável para tomada de decisão de direção da carga de treinamento.

Outra ferramenta, comumente utilizada como biomarcador de recuperação e fadiga, é a avaliação da altura de salto com contramovimento (SCM), que reflete o desempenho de força explosiva (TWIST \& HIGHTON, 2013). O desempenho do SCM é influenciado diretamente pelo ciclo de alongamento-encurtamento (CAE), que, por sua vez, pode ser afetado pela fadiga. Essa redução de desempenho em decorrência da fadiga observada no CAE pode ser afetada por modificações agudas em fatores centrais e periféricos, mas também pode ser influenciado por fatores estruturais, sendo mostrado que, de 24 a 72 horas após a última sessão de treinamento, pode estar associado a processos inflamatórios ligados ao dano muscular (CLAUDINO et al., 2012; KOMI, 2000; NICOL et al., 2006). Nesse sentido, estudos prévios investigaram a 
utilização dessa variável como uma ferramenta de monitoramento em diversos esportes e verificaram que as medidas relacionadas ao desempenho do SCM se mostraram eficazes para identificar variações no desempenho após os jogos durante períodos competitivos (CORMACK et al., 2008; OLIVER et al., 2015; RONGLAN et al., 2006; SPITERI et al., 2013).

Com explicitado acima, a literatura tem considerado a VFC como um possível marcador de recuperação e o SCM como um marcador de desempenho afetado pela fadiga. Mas, no processo de monitoramento, necessita-se de uma variável que registre a variação da carga percebida pelos atletas ao longo do tempo, sendo que o monitoramento da carga de treinamento acontece através da mensuração da carga interna e/ou da carga externa. A carga externa pode ser entendida como o trabalho completado pelo atleta medido independentemente da sua característica interna (WALLACE et al., 2009). Já a carga interna de treinamento é representada pelo estresse fisiológico imposto ao atleta e pode ser avaliada através do produto da Percepção Subjetiva de Esforço da sessão de treinamento pela duração total da sessão de treinamento, em minutos (FOSTER, 1998).

Contudo, a literatura não demonstrou até o momento como deveriam ser as tomadas de decisão a partir de oscilação dessas variáveis de monitoramento da carga ao longo de uma temporada competitiva com o objetivo de manter o desempenho próximo do máximo na maior parte do tempo. Dessa forma, o objetivo do presente estudo é, a partir de uma análise observacional de uma temporada competitiva de uma equipe de basquetebol profissional, demonstrar o comportamento longitudinal das variáveis, suas respectivas associações e a dinâmica de análise em um cenário real do esporte de alto rendimento. Com base nestes dados, pretende-se propor o nível de influência das variáveis de carga e recuperação no desempenho (SCM) e propor um modelo dinâmico de interação entre o monitoramento da carga e o planejamento das atividades em longo prazo.

\section{Método}

\section{Sujeitos}

A amostra deste estudo foi composta por 10 atletas profissionais de basquetebol do sexo masculino (idade, 27,9 \pm 5,3 anos; altura, 196,0 \pm 8,7 cm; massa corporal 96,3 $\pm 15,7 \mathrm{~kg}$; percentual de gordura, $9,7 \pm 2,8 \%$ ), que disputaram o principal campeonato da modalidade no Brasil.

\section{Desenho Experimental}

Os atletas passaram por uma pré-temporada de 14 semanas, sendo que a temporada completa teve uma duração de 34 semanas, consistindo em treinamento técnico-tático e treinamento físico. Durante o estudo os atletas realizaram saltos com contramovimento (SCM) antes do primeiro treino da semana e mediram a VFC três vezes por semanas antes do primeiro treino do dia. Além disso, após o início da temporada competitiva, os atletas voltavam a treinar aproximadamente 48 horas após os jogos, sendo realizadas as medidas sempre a partir deste 
momento.

Sessões de treinamento técnico-tático ocorreram uma ou duas vezes por dia, com média de nove sessões por semana. Enquanto as sessões de treinamento físico ocorreram uma vez por dia, com média de cinco sessões por semana. As medidas de percepção de recuperação e esforço foram realizadas antes e após o término de cada sessão de treinamento respectivamente, bem como o registro da duração de cada sessão. Os atletas estavam familiarizados com todos os procedimentos.

\section{Procedimentos}

Carga Interna - A percepção subjetiva de esforço da sessão (PSEs) (FOSTER, 1998) é mensurada através de uma escala que abrange o intervalo de 0 a 10 , sendo o número 0 associado ao repouso, e o número 10 associado ao esforço máximo. A partir desse resultado, a carga interna de treinamento foi calculada, determinada pelo produto entre o valor indicado na escala e a duração do treinamento em minutos. O valor resultante foi expresso em unidades arbitrárias. Dessa forma, as unidades arbitrárias de cada sessão de treinamento foram registradas e, ao final da semana, as cargas de cada unidade de treino foram somadas, obtendo-se a carga interna total semanal.

Salto com contramovimento (SCM) - Para medir a potência muscular dos membros inferiores, o SCM foi realizado em uma placa de contato denominada Plataforma Jumptest ${ }^{\circledR}$ (Hidrofit Ltda, Brasil) de 50x60cm, conectada ao software Multisprint ${ }^{\circledR}$ (Hidrofit Ltda, Brasil). Os atletas fizeram 3 tentativas com pausa de 15 segundos entre elas, registrando a altura de cada SCM. A análise dessa variável foi realizada pelo maior valor alcançado entre os três saltos. $\mathrm{O}$ atleta posicionou-se sobre o tapete de contato com os pés paralelos, com as mãos apoiadas na crista ilíaca, a cabeça para cima e o olhar para frente. Os atletas foram instruídos a não retirar a mão dessa posição a qualquer momento durante o salto e que os joelhos deveriam ser estendidos durante a fase de voo. Ao ouvir o comando "saltar", o indivíduo flexionou o joelho na medida em que ele se sentiu mais confortável e saltou imediatamente para cima o mais alto possível. Durante o teste foram considerados apenas saltos que atendiam a esses requisitos.

Variabilidade da Frequência Cardíaca (VFC) - As medidas da VFC foram realizadas antes da primeira sessão de treinamento. Os voluntários permaneceram deitados em decúbito dorsal e foram instruídos a permanecer calmos e quietos com respiração espontânea e com mínimo movimento corporal. Um cardiofrequencímetro (Polar® RS810i, Finlândia) foi utilizado para aquisição de dados. Os dados foram exportados para o Polar Precision Performance Software (Polar Electro Oy) e os intervalos RR foram exportados em formato ASCII para posterior análise. Os intervalos RR foram analisados no software de análise Kubios HRV, versão 2.0 (Biosignal Analysis e Medical Imaging Group - Universidade de Kuopio, Kuopio, Finlândia). Todos os dados foram inspecionados visualmente para remover batimentos ectópicos e, quando identificados, o filtro "low" do software Kubios foi utilizado (os intervalos 
analisados não tinham mais do que 3\% da seção removida) (TARVAINEN et al., 2014). Essas seções removidas manualmente foram substituídas pela interpolação dos intervalos RR adjacentes (TASK FORCE, 1996).

A variável da VFC analisada no domínio do tempo foi a raiz quadrada da soma dos quadrados dos intervalos RR adjacentes (simbolizada pela sigla RMSSD - Figura 1). Foi realizado um registro de 5 minutos sendo que os últimos 2 minutos foram utilizados para análise (BUCHHEIT, 2014). Para análise ao longo das semanas, a média das 3 gravações foi calculada com base em um estudo anterior que demonstrou ser a melhor maneira de realizar a análise (NAKAMURA et al., 2015).

\section{HRV Analysis Results}
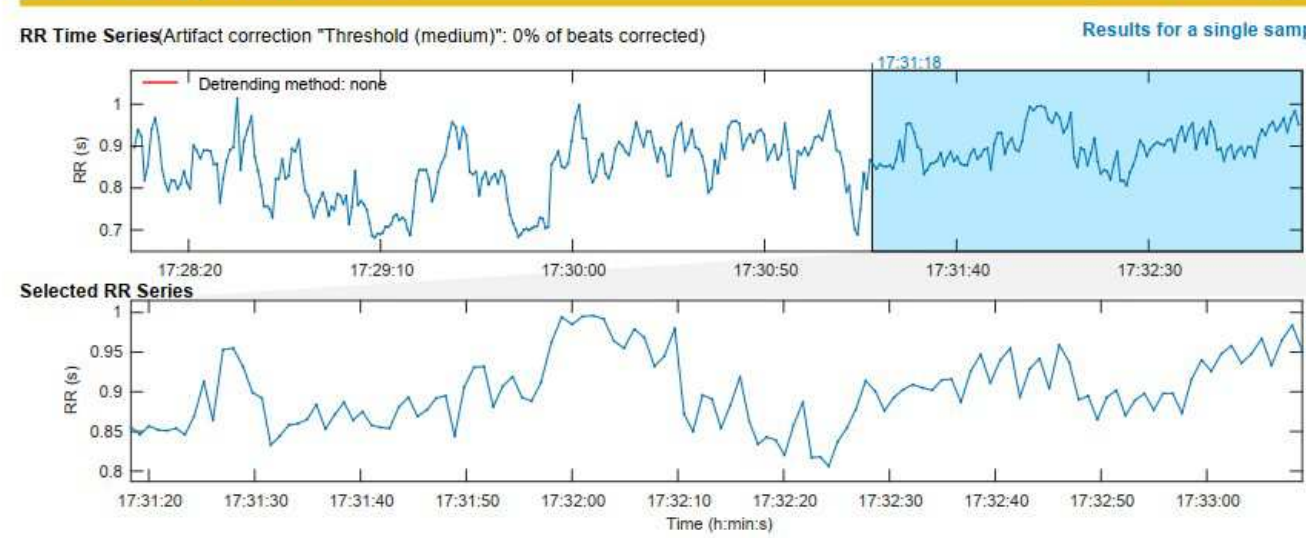
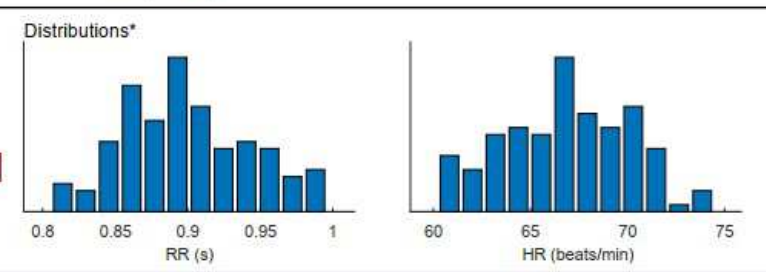

Figura 1 - Exemplo de Dashboard para análise da VFC no programa Kubios.

Percepção de Recuperação (PSR) - A percepção de recuperação era coletada diariamente através de uma escala que variava de 6 a 20, na qual o número 6 era ancorado pela frase "muito mal recuperado", e o 20, "muito bem recuperado".

Dinâmica do Monitoramento - A cada inserção de dado no sistema de monitoramento, o dashboard de cada atleta é atualizado para que os integrantes da comissão técnica e a equipe multidisciplinar possam acompanhar e avaliar o cenário atual de forma quase que instantânea, conforme o comportamento de cada atleta, ilustrado na Figura 2. O limite inferior (início da faixa vermelha, à esquerda de cada painel) se refere ao pior valor da variável no intervalo de tempo do "janelamento", neste caso, quatro semanas; a outra extremidade, verde, simboliza o 
melhor valor da variável no mesmo período; e a faixa central é o intervalo da Mínima Diferença Detectável (HOPKINS et al., 2009), onde não seria possível inferir melhoria ou piora do comportamento.
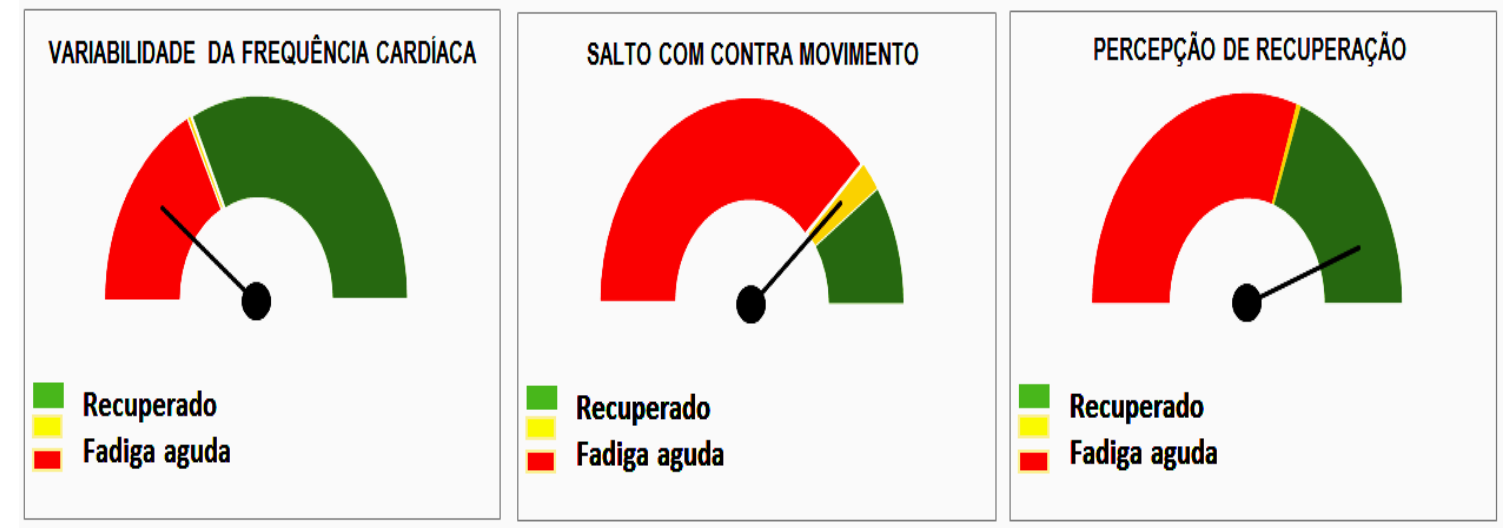

Figura 2 - Exemplo de dashboard individual da VFC, SCM e da PSR.

Com base na interação destas variáveis, somada ao comportamento da carga interna, a equipe técnica promoverá os ajustes necessários a cada circunstância que se apresenta.

\section{Análise Estatística}

Foi verificada inicialmente a normalidade e homocedasticidade dos dados obtidos através dos testes Shapiro-Wilk e Levene. Os dados foram descritos em média \pm desvio padrão. Todas as variáveis foram transformadas em percentual do seu valor máximo. A cada entrada de dado esse valor era atualizado em relação ao novo máximo, caso houvesse, em intervalos de 8 semanas. Foi realizada a correlação de Pearson para testar a associação entre as variáveis e uma análise de regressão múltipla utilizando como variável dependente o desempenho no SCM e como variáveis independentes os dados de carga interna, VFC, minutos jogados e percepção de recuperação. Adotou-se um nível de significância de $\mathrm{p} \leq 0,05$. Todos os dados foram plotados e analisados pelo pacote estatístico SPSS, versão 20.0.

\section{Resultados}

Todas as variáveis apresentaram normalidade e homocedasticidade $(\mathrm{p}>0,05)$. A Figura 3 apresenta o comportamento da carga interna total semanal e do tempo jogado total na semana. A Figura 4 apresenta o comportamento das variáveis de recuperação coletadas semanalmente (VFC, SCM PSR). 


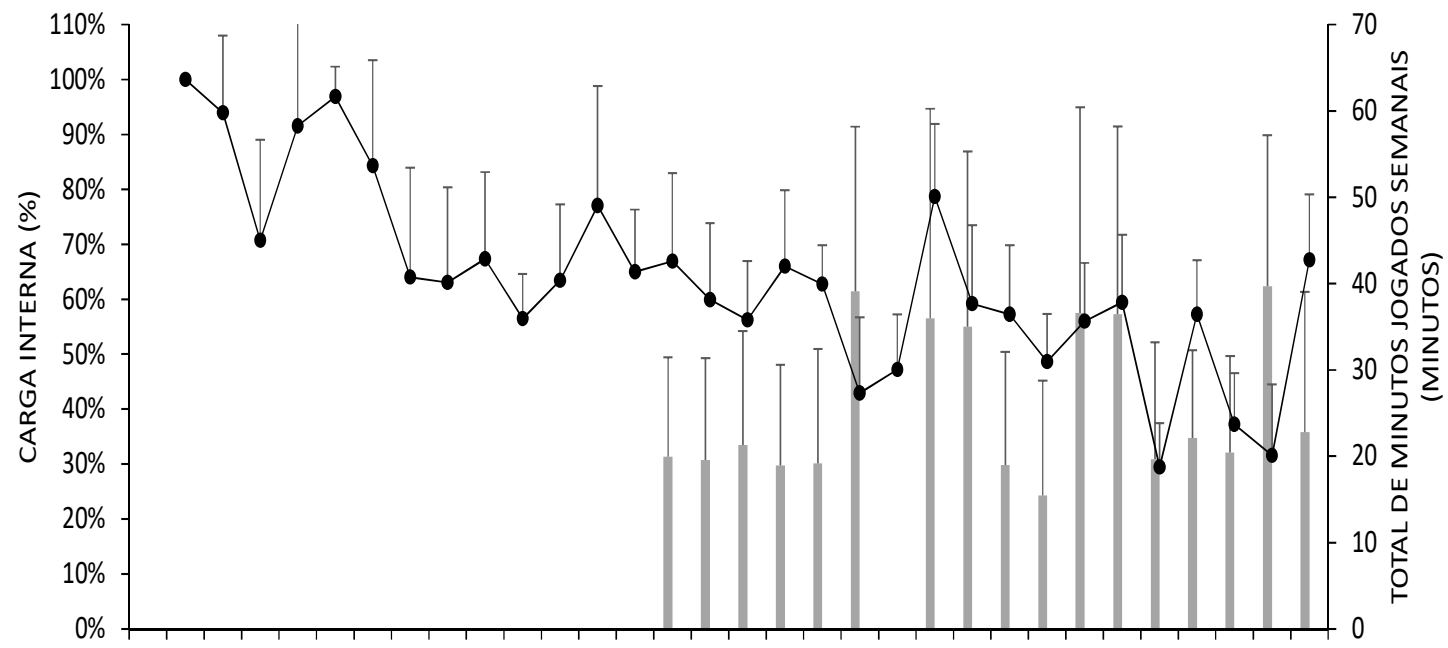

$122 \quad 3 \quad 4 \quad 5 \quad 6 \quad 7 \quad 8 \quad 9 \quad 1011121314151718192021222324252627293031323334$ Média de MINUTOS JOGADOS $\rightarrow-$ Média de \%Cl SEMANAS

Figura 3 - Variáveis de carga e tempo de jogo (média \pm desvio padrão).

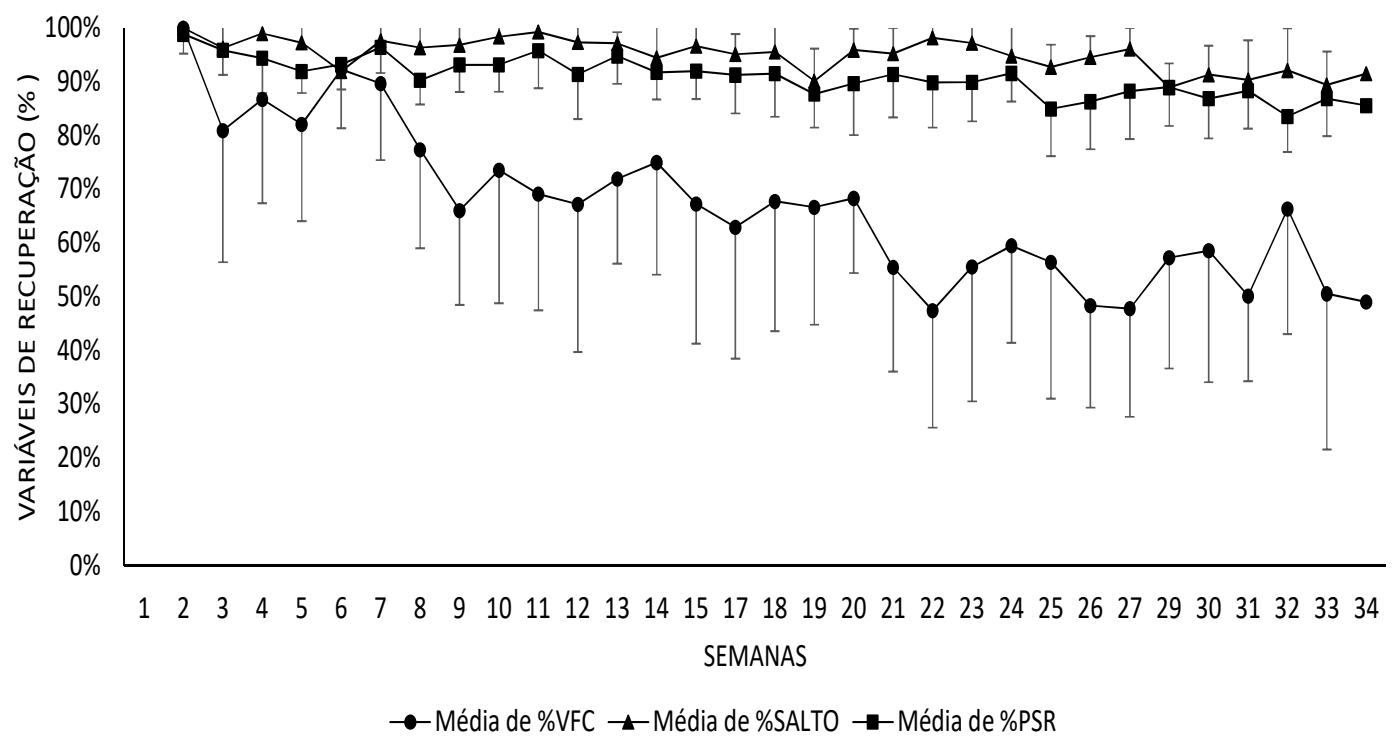

Figura 4 - Variáveis de recuperação ao longo do tempo (média \pm desvio padrão).

Tabela 1 - Correlação entre SCM e variáveis de carga e recuperação

\begin{tabular}{lcccc}
\hline Correlação & $\begin{array}{c}\text { Carga } \\
\text { Interna }\end{array}$ & $\begin{array}{c}\text { Minutos } \\
\text { Jogados }\end{array}$ & VFC & PSR \\
\hline $\boldsymbol{r}$ & $0,39 *$ & $-0,34$ & $0,74^{*}$ & $-0,08$ \\
$p$ & 0,02 & 0,12 & 0,001 & 0,62 \\
& & & & \\
\hline Fonte: Próprio autor $* \mathrm{p}<0,05$ & &
\end{tabular}

Fonte: Próprio autor. *p $<0,05$. 
A regressão múltipla apresentou um modelo com $\mathrm{r}^{2}$ ajustado de $0,65(\mathrm{p}=0,02)$, sendo que o desempenho de salto foi predito pela VFC e a carga interna. O modelo apresentou uma constante $(\beta 0=0,42)$ e seus respectivos $\beta 1$ e $\beta 2($ VFC $=1,132$; CARGA INTERNA $=-0,382)$. A variável PSR e minutos jogados foram excluídas do modelo por não apresentarem significância $(\mathrm{p}=0,37)$. Dessa forma, para cada aumento percentual da VFC, a variável SCM aumentaria 1,13 vez. Já em sentido contrário, para cada aumento de uma unidade percentual de carga interna a variável SCM reduziria em 0,38 ponto percentual.

A partir dos dados apresentados na regressão múltipla, foi proposto um modelo de monitoramento da carga no basquetebol que contempla a interação entre as variáveis e seu nível de interação (Figura 5). Dessa forma a carga interna deverá ser ajustada a partir da resposta da VFC, contrachecado pela resposta do SCM. Neste modelo a VFC seria o sinal para alteração da carga, e o teste de SCM a medida de desempenho para verificação da necessidade de mudança ou não da característica da carga. Não encontramos evidências fortes para considerar a PSR e os minutos jogados no modelo. Contudo, este efeito pode ser devido a limitações amostrais e circunstanciais, que necessitam de maiores investigações.

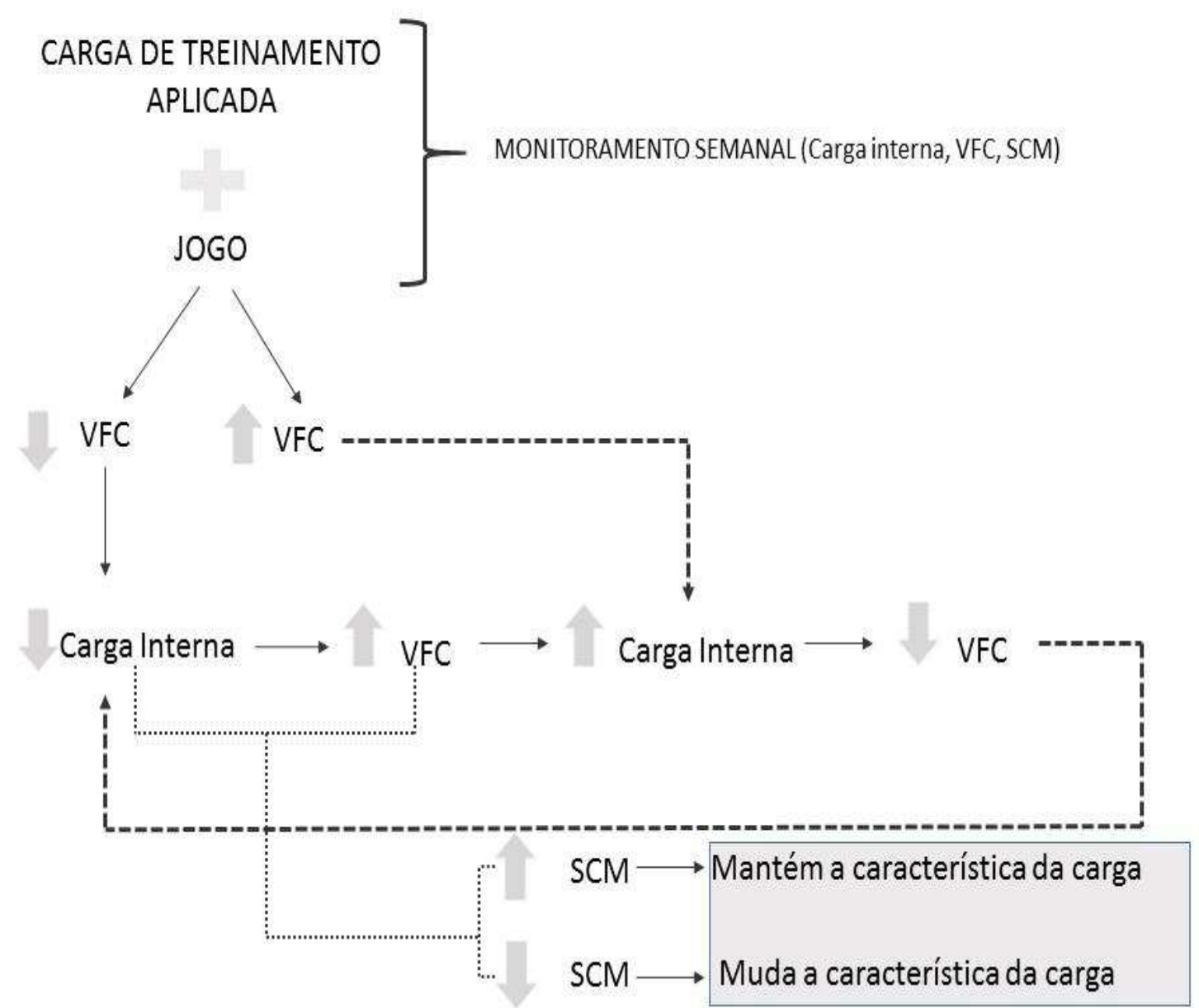

Figura 5 - Modelo dinâmico de monitoramento da carga de treinamento no basquetebol. VFC: Variabilidade da Frequência Cardíaca; SCM: Salto com contramovimento. 


\section{Discussão}

O objetivo do presente estudo foi avaliar o nível de influência das variáveis de carga e recuperação no desempenho do SCM e propor um modelo dinâmico de interação e análise entre o monitoramento da carga e o planejamento em longo prazo. A hipótese era que o desempenho no SCM sofreria alterações significativas a partir das variações de carga e recuperação, atingindo valores maiores nas semanas com menor carga e tempo de jogo e maior VFC e PSR. A nossa hipótese foi confirmada, mas foram excluídas do modelo de influência o tempo de jogo e a PSR. A primeira por parecer excessivamente generalista para refletir o desgaste individual; e a segunda, pela limitação do próprio instrumento.

Esperava-se que o tempo de jogo seria uma variável que influenciaria na altura do SCM devido ao efeito da fadiga pós-jogo para o atleta que jogou mais tempo. Contudo, resultados inconsistentes são relatados na literatura para a altura do SCM após os jogos durante períodos competitivos de diversos esportes, visto que alguns autores não encontraram diferenças significativas para esta variável nesta fase (GIBSON et al., 2016; SPITERI et al., 2013), enquanto outros autores observaram um decréscimo na altura do SCM (DELEXTRAT et al., 2012; OLIVER et al., 2015; RONGLAN et al., 2006). O mesmo acontece com o tempo necessário para a recuperação do desempenho neuromuscular após uma partida de basquetebol, já que Pliauga et al. (2015) demonstraram em jogadores universitários que jogar uma partida completa de basquetebol pode provocar alterações negativas na altura do SCM 24 horas após o término, o que não foi observado 48 horas após os jogos. Por outro lado, Chatzinikolaou et al. (2014) mostraram uma redução no desempenho da altura do SCM 48 horas após o jogo. Diante disso, o desempenho neuromuscular pode ser afetado depois de um jogo de basquetebol por diferentes períodos, conforme demonstrado na literatura e elucidada a tendência revelada no presente estudo através da correlação negativa, porém o tempo necessário para a recuperação total ainda é incerto e multifatorial, exigindo futuras investigações para esclarecer esse processo.

Apesar da inconsistência dos estudos em demonstrar a utilização do SCM como marcador de fadiga e recuperação, esta medida é consolidada na literatura enquanto ferramenta de controle em longo prazo do desempenho de potência neuromuscular, sendo uma variável de desempenho específica dentro do contexto de jogo do basquetebol (BEN ABDELKRIM et al., 2007; PLIAUGA et al., 2015). Dessa forma, o presente estudo avança dentro das informações contidas na literatura ao demonstrar que maiores valores de VFC levam a aumentos do desempenho de SCM. A VFC mensurada através do parâmetro RMSSD (que mensura a variância do intervalo R-R) é descrita na literatura como a "medida mais confiável e aplicável para o monitoramento diário" (PLEWS et al., 2013), sendo linearmente associada ao overreaching não funcional (BUCHHEIT, 2014). Contudo, alguns autores argumentaram da 
possibilidade de a VFC ser um indicador de fadiga geral (SCHMITT et al., 2015). Nesse sentido, um estudo com nadadores demonstrou que as mudanças na VFC podem estar associadas à percepção da qualidade do sono, fadiga, estresse ou humor, mas pouco associada à dor muscular tardia. Além disso, este estudo verificou que essa associação é variável entre indivíduos, demonstrando que alterações perceptivas influenciam de formas diferentes a resposta autonômica (FLATT et al., 2018). Portanto, o presente estudo demonstra que o monitoramento da "fadiga geral" pode auxiliar na manutenção de um elevado desempenho de potência.

O presente estudo também fortalece a hipótese de que elevadas cargas gerais de treinamento podem estar associadas a reduções do desempenho de potência. Foi demonstrado na literatura que a carga interna mensurada através do produto entre PSEs e duração do treinamento é um instrumento sensível para averiguar diferenças no volume e na intensidade dos treinamentos durante o período competitivo no basquetebol (AOKI et al., 2016). Assim, semanas com menor carga interna poderiam representar uma menor fadiga acumulada no momento pré-jogo, ocasionando uma menor queda no desempenho do SCM após o jogo. Markwick (2015) não encontrou uma relação entre a carga interna medida através de PSEs com o desempenho em saltos verticais durante uma temporada competitiva de 27 semanas com atletas profissionais da primeira divisão australiana de basquetebol. Nesta investigação, contudo, demonstramos a associação inversa entre as variáveis. Este efeito pode ter ocorrido devido a transformação das variáveis em percentual do máximo, que pode ter reduzido a variância intramostral, e permitido uma melhor associação entre as variáveis. Além disso, usamos a carga interna considerando também o volume (tempo) das atividades que originaram as PSEs.

É importante ressaltar as diferentes demandas de cada posição no jogo, conforme descrito por Svilar et al. (2018), que observou diferentes cargas internas e externas para as diferentes posições durante a temporada competitiva de atletas profissionais de basquetebol, evidenciando uma maior quantidade de ações em alta intensidade em armadores e alas do que em pivôs. Outros autores também já demonstraram essa relação através da análise de medidas de tempo durante os jogos (BEN ABDELKRIM et al., 2007; TORRES-RONDA et al., 2016). As diferentes demandas durante a partida podem influenciar a resposta de recuperação após o jogo. Segundo Ben Abdelkrim et al. (2007), as respostas de frequência cardíaca são significativamente menores durante o segundo e o quarto período do jogo, salientando uma menor quantidade de atividades em alta intensidade durante essas fases da partida. Diante disso, jogadores que jogam uma maior quantidade de minutos durante o segundo e o quarto período podem realizar cargas internas e externas diferentes de jogadores com mais minutos jogados durante o primeiro e o terceiro período. Portanto, a não associação entre a quantidade de minutos jogados durante a partida e a alteração do desempenho pode ter sido influenciada pelas 
diferentes demandas proporcionadas pela dinâmica do jogo e posições dos jogadores.

\section{Conclusão}

A partir dos dados apresentados neste estudo, de que a VFC e a Carga Interna podem influenciar o desempenho de salto, foi proposto um modelo dinâmico de monitoramento da carga (Figura 5). Neste modelo a VFC seria o sinal para alteração da carga, e o teste de SCM a medida de desempenho para verificação da necessidade de mudança ou não da característica da carga. O nosso estudo não encontrou evidências de que as respostas de percepção de recuperação e o tempo jogado podem auxiliar na manutenção ou evolução do desempenho de potência. Sendo assim, treinadores e preparadores físicos podem utilizar dados fisiológicos (VFC), perceptivos (CI) e neuromusculares (SCM) para ajustar o planejamento de equipes de basquetebol de alto rendimento e garantir que os atletas estejam no melhor nível de desempenho em momentos decisivos.

\section{Referências}

AOKI, M. S. et al. Monitoring training loads in professional basketball players engaged in a periodized training program. The Journal of Strength \& Conditioning Research, v. 31, n. 2 , p. 348-358, 2017.

BELLENGER, C. R. et al. Monitoring Athletic Training Status Through Autonomic Heart Rate Regulation : A Systematic Review and Meta-Analysis. Sports Medicine, v. 46, n. 10, p. 14611486, 2016.

BEN ABDELKRIM, N. et al. Time-motion analysis and physiological data of elite under-19year-old basketball players during competition * Commentary. British Journal of Sports Medicine, v. 41, n. 2, p. 69-75, fev. 2007.

BUCHHEIT, M. Monitoring training status with HR measures: Do all roads lead to Rome? Frontiers in Physiology, v. 5, p. 1-19, 2014.

BUCHHEIT, M.; LAURSEN, P. B.; AHMAIDI, S. Parasympathetic reactivation after repeated sprint exercise. American journal of physiology. Heart and circulatory physiology, v. 293, n. 1, p. H133-H141, 2007.

CARDINALE, Marco; VARLEY, Matthew C. Wearable training-monitoring technology: applications, challenges, and opportunities. International journal of sports physiology and performance, v. 12, n. Suppl 2, p. S2-55-S2-62, 2017.

CASTAGNA, C. et al. Validity of an On-Court Lactate Threshold Test in Young Basketball Players. Journal of Strength and Conditioning Research, v. 24, n. 9, p. 2434-2439, set. 2010.

CATALDO, A. et al. Influences of baseline heart rate variability on repeated sprint performance in young soccer players. The Journal of Sports Medicine and Physical Fitness, v. 56, n. 4, p. 491-6, 2016.

CHATZINIKOLAOU, A. et al. The microcycle of inflammation and performance changes after a basketball match. Journal of Sports Sciences, v. 32, n. 9, p. 870-882, maio 2014.

CLAUDINO, J. et al. Pre Vertical Jump Performance to Regulate the Training Volume. International Journal of Sports Medicine, v. 33, n. 02, p. 101-107, fev. 2012.

CORMACK, S. J. et al. Neuromuscular and Endocrine Responses of Elite Players During an Australian Rules Football Season. International Journal of Sports Physiology and Performance, v. 3, p. 439-453, 2008. 
DELEXTRAT, A.; TROCHYM, E.; CALLEJA-GONZÁLEZ, J. Effect of a typical in-season week on strength jump and sprint performances in national-level female basketball players. The Journal of sports medicine and physical fitness, v. 52, n. 2, p. 128-36, abr. 2012.

FLATT, A. A.; ESCO, M. R.; NAKAMURA, F. Y. Association between Subjective Indicators of Recovery Status and Heart Rate Variability among Division-1 Sprint-Swimmers. Sports, v. 6, n. 93, p. 1-9, 2018.

FOSTER, C. Monitoring training in athletes with reference to overtraining syndrome. Medicine and science in sports and exercise, v. 30, n. 7, p. 1164-8, jul. 1998.

FOSTER, C.; RODRIGUEZ-MARROYO, J. A.; DE KONING, J. J. Monitoring training loads: the past, the present, and the future. International journal of sports physiology and performance, v. 12, n. Suppl 2, p. S2-2-S2-8, 2017.

GABBETT, T. J. The training-injury prevention paradox: should athletes be training smarter and harder?. Br J Sports Med, v. 50, n. 5, p. 273-280, 2016.

GIBSON, N. E.; BOYD, A. J.; MURRAY, A. M. Countermovement Jump is Not Affected During Final Competition Preparation Periods in Elite Rugby Sevens Players. Journal of Strength and Conditioning Research, v. 30, n. 3, p. 777-783, mar. 2016.

HEIDARI, J. et al. Multidimensional monitoring of recovery status and implications for performance. International Journal of Sports Physiology and Performance, p. 1-24, 2018.

HOFFMAN, J. R. et al. A Comparison Between the Wingate Anaerobic Power Test to Both Vertical Jump and Line Drill Tests in Basketball Players. The Journal of Strength and Conditioning Research, v. 14, n. 3, p. 261, 2000.

HOPKINS, W. G. et al. Progressive statistics for studies in sports medicine and exercise science. Medicine and Science in Sports and Exercise, v. 41, n. 1, p. 3-12, 2009.

KOMI, P. V. Stretch-shortening cycle: a powerful model to study normal and fatigued muscle. Journal of Biomechanics, v. 33, p. 1197-1206, 2000.

LEE, E. C. et al. Biomarkers in sports and exercise: Tracking health, performance, ande recovery in athletes. Journal of strength and conditioning research, v. 31, n. 10, p. 29202937, 2017.

MARKWICK, W. Training load quantification in professional Australian basketball and the use of the reactive strength index as a monitoring tool. [s.l: s.n.]. 2015

MIZUNO, K. et al. Mental fatigue caused by prolonged cognitive load associated with sympathetic hyperactivity. Behavioral and brain functions, v. 7, n. 17, p. 1-7, 2011.

MIZUNO, K. et al. Fatigue correlates with the decrease in parasympathetic sinus modulation induced by a cognitive challenge. Behavioral and brain functions, v. 10, n. 1, p. 25, 2014.

NAKAMURA, F. Y. et al. Alteração do limiar de variabilidade da freqüência cardíaca após treinamento aeróbio de curto prazo. Motriz, v. 11, n. 1, p. 1-9, 2005.

NAKAMURA, F. Y. et al. Ultra-Short-Term Heart Rate Variability is Sensitive to Training Effects in Team Sports Players. Journal of sports science \& medicine, v. 14, n. 3, p. 602-605, 2015.

NAKAMURA, F. Y. et al. Monitoring weekly heart rate variability in futsal players during the preseason: the importance of maintaining high vagal activity. Journal of Sports Sciences, v. 34, n. 24, p. 2262-2268, 2016.

NARAZAKI, K. et al. Physiological demands of competitive basketball. Scandinavian Journal of Medicine \& Science in Sports, v. 19, n. 3, p. 425-432, jun. 2009.

NICOL, C.; AVELA, J.; KOMI, P. V. The stretch-shortening cycle : a model to study naturally occurring neuromuscular fatigue. Sports medicine, v. 36, n. 11, p. 977-99, 2006.

OLIVER, J. L.; LLOYD, R. S.; WHITNEY, A. Monitoring of in-season neuromuscular and perceptual fatigue in youth rugby players. European Journal of Sport Science, v. 15, n. 6, p. 514-522, ago. 2015. 
PANISSA, V. L. G. et al. High-intensity intermittent exercise and its effects on heart rate variability and subsequent strength performance. Frontiers in Physiology, v. 7, p. 1-7, 2016.

PLEWS, D. J. et al. Training Adaptation and Heart Rate Variability in Elite Endurance Athletes : Opening the Door to Effective Monitoring. Sports Medicine, v. 43, n. 9, p. 773-781, 2013.

PLIAUGA, V. et al. The Effect of a Simulated Basketball Game on Players' Sprint and Jump Performance, Temperature and Muscle Damage. Journal of human kinetics, v. 46, p. 167-75, jun. 2015.

RONGLAN, L. T.; RAASTAD, T.; BORGESEN, A. Neuromuscular fatigue and recovery in elite female handball players. Scandinavian Journal of Medicine and Science in Sports, v. 16, n. 4, p. 267-273, ago. 2006.

SCHMITT, L.; REGNARD, J.; MILLET, G. P. Monitoring Fatigue Status with HRV Measures in Elite Athletes : An Avenue Beyond RMSSD ? Frontiers in Physiology, v. 6, p. 1-3, 2015.

SPITERI, T. et al. Monitoring neuromuscular fatigue in female basketball players across training and game performance. ECU Publications 2013, jan. 2013.

SVILAR, L. et al. Positional Differences in Elite Basketball: Selecting Appropriate Training Load Measures. International Journal of Sports Physiology and Performance, p. 1-24, jan. 2018.

TARVAINEN, M. P. et al. Kubios HRV - Heart rate variability analysis software. Computer Methods and Programs in Biomedicine, v. 113, n. 1, p. 210-220, 2014.

TASK FORCE. Heart rate variability - Standards of measurement, physiological interpretation, an clinical use. European Heart Journal, v. 17, p. 354-381, 1996.

TAYLOR, J. B. et al. Activity Demands During Multi-Directional Team Sports: A Systematic Review. Sports Medicine, v. 47, n. 12, p. 2533-2551, 2017.

TORRES-RONDA, L. et al. Position-Dependent Cardiovascular Response and Time-Motion Analysis During Training Drills and Friendly Matches in Elite Male Basketball Players. Journal of Strength and Conditioning Research, v. 30, n. 1, p. 60-70, jan. 2016.

TWIST, C.; HIGHTON, J. Monitoring fatigue and recovery in rugby league players. International journal of sports physiology and performance, v. 8, n. 5, p. 467-74, set. 2013.

VESTERINEN, $V$. et al. Individual endurance training prescription with heart rate variability. Medicine and science in sports and exercise, v. 48, 2016.

WALlACE, L. K.; SLATTERY, K. M.; COUTTS, A. J. The Ecological Validity and Application of the Session-RPE Method for Quantifying Training Loads in Swimming. Journal of Strength and Conditioning Research, v. 23, n. 1, p. 33-38, jan. 2009.

Artigo recebido em: 26/06/2019

Artigo aceito para publicação em: 09/09/2019 